P113 (continued)

Results: Four typologies of parents were observed: Supportive, Negative, Laid-back, and Mixed. Parents with Negative and Supportive typologies had more affluent kitchen equipment whereas parents with Laid-back and Mixed typologies had less affluent kitchen equipment $(\mathrm{p}=0.014)$. Parents with Negative typologies had the least cluttered kitchen environments; those with Mixed typologies had the most cluttered environments $(\mathrm{p}<$ 0.000). Youth whose parents were Laid-back or Mixed typology asked for assistance more often than those with Supportive or Negative typology parents $(\mathrm{p}<0.000)$. Youth of parents with Supportive typology had the highest levels of cooking confidence whereas youth of parents with Laid-back typology had the lowest levels of cooking confidence $(\mathrm{p}=0.001)$.

Conclusion and Implications: Cooking-related parenting typologies were uniquely identified in this research. Impact of differing parenting typology on youth health-related behavior warrants further investigation.

Funding: USDA

\section{P114 Impact of a Mindfulness-Based Program on Child Diet and Eating Behaviors}

Lynn Brann, PhD, RD, FAND, lbrann@syr.edu, Syracuse University, 559 White Hall, Syracuse, NY 13244;

Rachel Razza, PhD, Syracuse University; Dessa BergenCico, PhD

Objective: To examine the impact of a mindfulnessbased program (MBP) on child dietary intake and eating behaviors.

Study Design, Setting, Participants, Intervention: A six-week mindful eating and yoga intervention to promote children's self-regulation was conducted in two childcare centers (intervention $n=24$; control $n=20$ ) with children 3-5 years old.

Outcome Measures and Analysis: Pre- and post-intervention, parents completed three-day child food records and the Child Eating Behavior Questionnaire (CEBQ) to assess factors such as food responsiveness and satiety responsiveness. Children's Eating in the Absence of Hunger (EAH) was measured pre- and post-intervention. Outcome evaluation analyses included paired t-tests, McNemar tests, independent samples t-tests and Mann Whitney U tests.

Results: Children's diets were low in key nutrients of concern including fiber, vitamin $\mathrm{D}$, vitamin $\mathrm{E}$ and potassium and were high in sodium. The intervention group had significantly higher intakes of several nutrients compared to the intervention group at the pre-assessment; however, both groups consumed below recommendations for vitamin $\mathrm{D}$ and potassium and above for sodium. No significant difference were found between groups for dietary intake at the post-assessment. There were no significant differences in the subscales of the CEBQ between the intervention and control groups at the pre- and post-assessment. After hunger was neutralized, there were no differences in the number of calories consumed within or between groups of children for EAH.

Conclusions and Implications: This pilot study did not show a significant impact of utilizing a MBP on child dietary intake and eating behaviors. Further research needs to evaluate success of MBPs with a larger sample size and greater involvement of parents is necessary.

Funding: Falk College Research Center at Syracuse University

\section{P115 Increasing Children's Fruit and Vegetable Consumption Using Nutrition Education and Active Choice Principles}

Robyn M. Cafiero, MS, robyncafiero@gmail.com, Montclair State University, 253 Park Avenue, Rutherford, NJ 07070; Yeon Bai, PhD, RD, Montclair State University; Doreen Liou, EdD, RD; Charles Feldman, PhD

Objective: To increase children's fruit and vegetable consumption using nutrition education and active choice principles.

Study Design, Setting, Participants, Intervention: The study used pre-/post-intervention comparison design. The intervention utilized a dual module of the Social Cognitive Theory (SCT) and active choice (AC) for second graders in one school in Northern, NJ. For nine months, the intervention group received a combination of nutrition education and AC; the control group received AC. AC allowed the participants to choose between two fruits and/or vegetables.

Outcome Measures and Analysis: Variables measured included fruit/vegetable preference, consumption, reciprocal determinism, self-efficacy, behavioral capability, and modeling from SCT. Paired/independent t-tests, chisquare analyses were employed to compare groups.

Results: Eighty-nine students participated in the study (intervention $=46$, control $=43$ ): $57 \%$ female and $43 \%$ males; mean age 7.64 (SD 0.48). No change was observed in fruit consumption. Vegetable consumption and preference differed after intervention between groups: for consumption (self-efficacy), 6.5\% increase for intervention and $4.5 \%$ decrease for control $(\mathrm{p}=.01)$; for preference, $4.3 \%$ increase for intervention and $2.4 \%$ decrease for control $(\mathrm{p}=.57)$. Similarly, knowledge (behavior capability) of fruit/vegetables increased $4.3 \%$ in intervention, but decreased $2.4 \%$ in control $(\mathrm{p}=.14)$. All students were engaged within AC even if they did not take a fruit/vegetable that day.

Conclusions and Implications: Nutrition education combined with AC had a positive impact on knowledge and vegetable consumption/preference in the intervention group. A longer invention duration with multiple locations may grow the study validity for innovative program to be implemented throughout school districts nationwide.

Funding: Jennifer Layne Acupuncture and Wellness; ShopRite of Rochelle Park, NJ 